\title{
22. Territoire en questions
}

\author{
(C) Springer-Verlag France 2010
}

\section{1}

\section{Perceptions, appropriations et représentations des territoires de vie des $\mathbf{7 5}$ ans et plus \\ B. Nader}

Université Paris-Est, Créteil, LAB-URBA, France

Le territoire de vie des aînés est un espace vécu, approprié et ressenti différemment en fonction de leur âge, de leur sexe, de leurs conditions socioéconomiques, de leur état de santé, de leur histoire personnelle mais également des caractéristiques écologiques du quartier dans lequel ils habitent. L'étude des pratiques spatiales des 75 ans et plus permet de mieux comprendre comment ils s'approprient leur territoire au quotidien et quels sont leurs besoins pour rester intégré dans la société et maintenir leur autonomie. Le territoire est à la fois le support des pratiques spatiales, le produit des politiques publiques et des aménagements urbains mais également le cœur des enjeux sociétaux d'intégration des aînés.

Une étude s'est déroulée dans le $14^{\mathrm{e}}$ arrondissement à Paris auprès de 158 personnes âgées de 75 à 102 ans, valides et autonomes, lors d'entretiens semi-directifs dans lesquels il leur était demandé de dessiner leur quartier sous forme de carte mentale. La perception de la qualité de vie au sein de leur territoire de vie et les déplacements au quotidien sont analysés et confrontés à la représentation de la carte mentale. Lorsque la perception de la qualité de vie est mauvaise, les liens sociaux sont souvent dégradés, les mobilités réduites dans l'espace et liées aux besoins essentiels du quotidien : l'appropriation du territoire est alors déclinante et emblématique d'un isolement social. À l'inverse, les personnes ayant une bonne perception de la qualité de vie ont un espace vécu plus étendu et des mobilités favorisées par leurs liens sociaux et leurs activités associées. Cette contribution présentera les différents types d'appropriation de l'espace des aînés et les besoins identifiés à échelle locale dans les différents quartiers étudiés.

\section{2}

\section{Dépression et qualité de l'environnement géographique de proximité : quelles corrélations ? \\ P-M. Chapon ${ }^{1}$ J. Gueslot ${ }^{2}$ \\ ${ }^{1}$ Université Lyon-III, EA 4129 et LAB-URBA, \\ ICADE (caisse des dépôts et consignations), FRANCE \\ ${ }^{2}$ Université Paris-Est, Créteil, France}

Peu de travaux étudient la corrélation entre un environnement géographique favorable ou défavorable et l'état dépressif de personnes âgées. C'est dans la perspective d'étudier ces relations, qu'un travail a été mené avec Mondial Assistance sur un échantillon de 713 personnes âgées équipées d'un dispositif de téléassistance sur l'ensemble du territoire métropolitain. Les données recueillies sont traitées en utilisant des méthodes d'analyses statistiques exploratoires afin de déterminer des profils de comportements spatiaux des personnes âgées. Une corrélation a pu être établie entre la qualité de l'environnement géographique (proximité de commerces, transports, services) et l'état de dépression des personnes enquêtées. Cependant, les aspects géographiques ne constituent qu'un premier élément.

Il s'est avéré également que les personnes âgées dont la famille était présente à proximité de leur lieu de résidence obtenaient des scores plus faibles aux questions sur l'état dépressif et ce, de manière significative. Nous démontrons ainsi l'importance du lien social de la personne âgée avec son cercle familial, et donc l'importance d'une proximité géographique entre les personnes âgées et leur famille ou encore leurs amis.

\section{3}

NTIC et redéfinition d'espaces sociaux sur un territoire S. Pennec, F. Le Borgne, G. Mendal Atelier de recherche sociologique (EA 3149), université de Bretagne occidentale, France

S'agissant des personnes du grand âge, les technologies sont généralement présentées sous des angles opposés. Tantôt elles apparaissent comme des sources potentielles d'exclusion et de difficultés accrues dans différents domaines (accès aux informations, aux administrations, aux transports, etc.). A contrario, elles sont aussi décrites comme des substituts possibles, réponses à des besoins considérés en premier lieu sous l'angle des déficits associés au vieillissement. Pour autant, « le maintien du lien social » est un objectif de plus en plus fréquemment énoncé et associé aux autres fonctions attendues. Dans ce domaine, les sciences sociales sont convoquées principalement pour rendre compte des spécificités des usages des techniques, concernant des projets parmi lesquels les objectifs de soins, d'assistance et de surveillance paraissent dominer lorsqu'il s'agit du public âgé. Le questionnement développé se trouve parfois réduit aux seules dimensions de l'objet construit par les concepteurs, depuis les sciences de l'ingénieur jusqu'aux divers promoteurs des services étudiés, l'application des sciences sociales de limitant à des enquêtes instrumentales là où s'avère nécessaire l'apport des analyses fondamentales.

Dans cette contribution, nous exposons plusieurs réalisations en matière de TIC, situées sur un territoire donné, pour chercher à rendre compte des logiques d'acteurs et de leurs politiques en direction de la population âgée. 